\title{
Modeling the barotropic response of the global ocean to atmospheric wind and pressure forcing - comparisons with observations
}

\author{
Loren Carrère and Florent Lyard \\ Laboratoire d'Etudes en Géophysique et Océanographie Spatiale, Toulouse, France
}

Received 17 October 2002; accepted 10 February 2003; published 19 March 2003.

[1] A global simulation of the ocean response to atmospheric wind and pressure forcing has been run during the Topex/Poseidon (T/P) period (1992-2002), using a new hydrodynamic finite element (FE) model, MOG2D-G. Model outputs are compared to in situ observations with tide gauge data (TG) and bottom pressure gauge data (BPR), and also with $\mathrm{T} / \mathrm{P}$ altimetric cross over points (noted CO). Intercomparisons were performed over the 1993-1999 period. The model correction reduces the sea level variance by more than $50 \%$ at TG locations, and by more than $15 \%$ at $\mathrm{T} / \mathrm{P} \mathrm{CO}$, when compared to the classical inverse barometer correction (IB). The model impact differs between high and low latitudes: in the very energetic high latitudes areas, MOG2D-G is efficient in reducing the variance, while at low latitudes, the results are similar to the IB static response. In shallow water, the model shows an oceanic response very different from the IB response. In conclusion, MOG2D-G models the high frequency (HF) atmospheric forced variability of the global ocean with unprecedented accuracy. INDEX TERMS: 4255 Oceanography: General: Numerical modeling; 4504 Oceanography: Physical: Air/sea interactions (0312); 4560 Oceanography: Physical: Surface waves and tides (1255); 4564 Oceanography: Physical: Tsunamis and storm surges. Citation: Carrère, L., and F. Lyard, Modeling the barotropic response of the global ocean to atmospheric wind and pressure forcing - comparisons with observations, Geophys. Res. Lett., 30(6), 1275, doi:10.1029/2002GL016473, 2003.

\section{Introduction}

[2] The T/P and Jason altimeters deliver very accurate data sets (within 2 centimeter global error for T/P). However for mesoscale circulation applications and satellite calibration campaigns, the HF ocean signal (periods less than 20 days for $\mathrm{T} / \mathrm{P}$ ), is aliased into the low frequency band (LF; periods larger than 20 days for $\mathrm{T} / \mathrm{P}$ ), and needs to be corrected from independent models at centimetric accuracy. The present HF tidal corrections have mainly reached this requirement, through the high resolution hydrodynamic model FES [Lefèvre et al., 2002] or the assimilated model GOT [Ray, 1999]. In contrast, the ocean response to meteorological forcing is still poorly accounted for by simply applying the inverted barometer correction (IB, [Wunsch and Stammer, 1997]).

[3] The classical IB approximation formulates the static response of the ocean to atmospheric pressure forcing, and wind effects are totally ignored. The validity of this IB assumption depends on the time and space scales considered: the ocean response to atmospheric pressure generally differs from the IB at periods $<3$ days and at high latitudes. Wind effects also prevail particularly around the 10 day period.

[4] Earlier barotropic ocean models have been developed by Ponte [1991], and Mathers [2000], characterised by a finite difference space discretisation on fairly large grids of $1^{\circ}$ by $1^{\circ}$. They include very strong dissipation processes, either via a strong linear and uniform bottom friction or a large horizontal eddy viscosity.

[5] The aim of our work is to model the HF oceanic response to meteorological forcing with a new realistic hydrodynamic model, with the best possible accuracy. We have performed a global simulation over the 10 year T/P period (1992-2002). We also estimate the relevance of applying such a numerical model correction to in-situ (TG and $\mathrm{BPR})$ and altimeter (T/P) observations.

\section{Methodology-Hydrodynamic Model MOG2D-G}

\subsection{Description}

[6] MOG2D-G (2D Gravity Waves model) is a barotropic, non linear and time stepping model, derived from (Lynch and Gray [1979]; Greenberg and Lyard, personal communication). The model governing equations are the classical shallow water continuity and momentum equations. The model can include tides and its main originality is a finite element space discretisation (FE), which allows us to increase the resolution in strong topographic gradient areas or in shallow seas.

[7] The FE mesh is shown in Figure 1. The grid size ranges from $400 \mathrm{~km}$ in deep ocean to $20 \mathrm{~km}$ in coastal, shallow areas. This medium resolution is a good compromise to resolve the physics and minimise the computational costs. The global simulation domain includes shallow water areas and marginal seas, which are generally neglected by other studies; including continental shelves, the Mediterranean Sea, Hudson Bay, Bering Strait, the Arctic Ocean and the Weddell and the Ross Seas, whose distinctive feature is to be partly covered with a permanent ice field. The ice cover impact is taken into account through the diminution of the water column height, due to the submerged ice thickness [Lyard, 1997]. The bottom friction is parameterised by a quadratic law. A new dissipation process is introduced to take into account the barotropic to baroclinic energy transfer via internal wave (IW) generation over topographic features. This term is well known to dissipate around $1 / 3$ rd of the global barotropic energy [Egbert and Ray, 2000]. $-C L \bar{N}(\vec{\nabla} H \cdot \vec{u}) \vec{\nabla} H$ is the internal wave gen- 


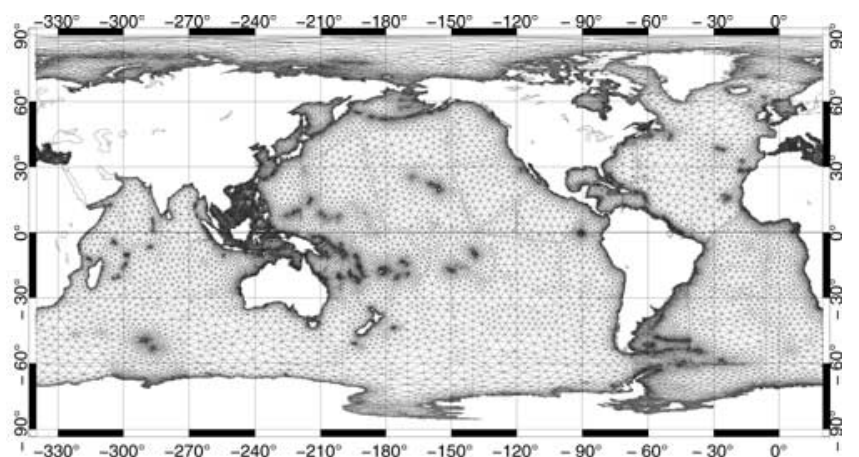

Figure 1. Mid-resolution finite element mesh used.

eration parameterisation. L is a tidal incursion scale; $\bar{N}$ is equivalent to a depth-averaged Brünt-Vässälä frequency [Baines, 1982]; $\mathrm{H}$ is the depth; $\mathrm{u}$ is the barotropic current; $\mathrm{C}$ is a tuning parameter. This parameterisation of the impact of an oscillating current on a sloping topography, has been deduced by analogy with the formulation of the effect of a permanent current flowing over an oscillating bathymetry [Gill, 1982].

[8] This formulation is complementary to those of Jayne and St. Laurent [2001] who dealt with roughness effects. A Smagorinsky viscosity scheme [Smagorinsky, 1963] takes into account the varying $\mathrm{FE}$ grid cells size; the mean viscosity coefficient is only $90 \mathrm{~m}^{2} / \mathrm{s}$.

[9] The semi-implicit numerical scheme combined with a new sub-step time process allows us to maintain a larger time step for most calculations, while preserving model stability. At the present time, the model is running with a 3 min time step.

\subsection{Validation}

[10] For validation purposes, tides were simulated with MOG2D-G, as an example of HF gravity waves, with similar dynamics to our atmospheric forced ocean waves. Oceanic tides are also well-known on the global ocean through observations (TG) and high precision numerical models (FES 99, GOT). MOG2D-G computed tides are of state of the art quality for non assimilating models. Tide gauge performances are given in Table 1; they are better than the FES 99 hydrodynamic model for the HF (M2 and $\mathrm{K} 1$ ); and equivalent to the FES 99 assimilated model performances for the LF waves (Mm, Mf). Note that including the IW generation parameterisation allows us to divide the bias/rms of the high frequency waves, M2 and $\mathrm{K} 1$, by a factor of 2 .

[11] The ocean response to an idealised forcing by a Rossby-Haurwitz westward propagating pressure wave at a period of 5 days (RH5) was also investigated. Comparing results with those of Ponte [1997] and Mathers [2000], shows very similar 5 days structures, which are far from a purely IB oceanic response. Given these tidal and RH5 computation results, we consider that the MOG2D-G model has been validated for both high and low frequencies.

\subsection{Forcing}

[12] Pressure and wind speeds (at the altitude of $10 \mathrm{~m}$ ) are taken from the ECMWF analysis fields [ECMWF, 1991]. These forcing fields have been interpolated onto a regular $1^{\circ}$ by $1^{\circ}$ grid. The temporal resolution is 6 hours, so
Table 1. Mean Bias/Rms for 95 Tidal Gauges From the WOCE and IAPSO Data Sets, for Mog2d and Fes99 Models, in cm; IW: Internal Wave Generation is Included; Assim.: Assimilated Model

\begin{tabular}{lllll}
\hline & \multicolumn{1}{c}{$M_{2}$} & \multicolumn{1}{c}{$K_{1}$} & \multicolumn{1}{c}{$M m$} & \multicolumn{1}{c}{$M f$} \\
\hline Mog2d & $4.7 / 8.4$ & $0.4 / 2.1$ & $-0.3 / 0.4$ & $-0.1 / 0.2$ \\
Fes99 & $4.6 / 13.1$ & $0.4 / 2.3$ & - & - \\
Mog2d + IW & $1.3 / 6$ & $0 / 1.4$ & - & - \\
Fes99 + assim. & $0.1 / 1.3$ & $0 / 1.1$ & $-0.3 / 0.4$ & $-0.1 / 0.2$ \\
\hline
\end{tabular}

frequencies lower than 12 hours are misrepresented. The forcing fields are linearly interpolated to the model time step.

[13] At each time step, the spatial average over the global ocean is removed from the atmospheric pressure, to make it consistent with the IB correction.

[14] The wind stress is deduced from wind speed with the classical formula of Hellereman and Rosenstein [1983]; the heat flux between ocean and the atmosphere is taken equal to zero. In essence, our barotropic model does not include any 3D dissipation; some unrealistically strong deep ocean circulation can appear when the annual mean wind stress is kept in the forcing. An additional dissipation term is thus introduced, giving a local increase in the quadratic friction coefficient, in order to parameterise the baroclinic dissipation between different ocean layers. Only the atmospheric induced currents are taken into account in this formulation.

[15] Initially, the ocean is at rest, then the forcing is established linearly during 3 days to prevent shocks. The simulation was performed over the T/P period November 1992-2002, including both atmospheric pressure and wind forcing. Since the model spin up takes less than 2 months, comparisons with tidal gauges and satellites observations have been performed on the 1993-1999 period. The pressure-only forcing configuration has been tested on the year 1995.

\section{Comparison to Tidal Gauges}

[16] The MOG2D-G solutions are compared to a data set of 142 TG (Figure 2), from the WOCE and MEDGLOSS databases [Ponchaut et al., 2001], to evaluate the quality of the model in coastal areas. The model correction is applied to the observed signal and, we measure the improvements with respect to a classical IB correction. We quantify the reduction of the residual variance for both the aliased frequency band ( 0.5 days to 20 days for $\mathrm{T} / \mathrm{P}$, referred to

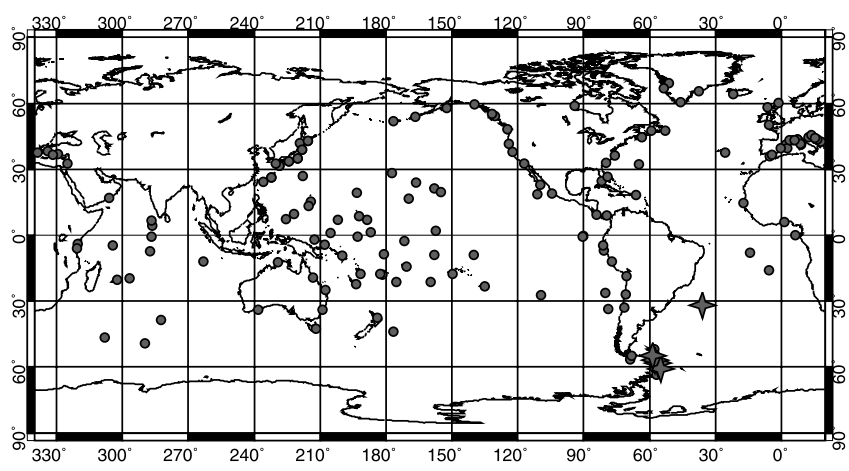

Figure 2. Localisation of all tidal gauges (circles) and bottom pressure gauges (stars) considered in this study. 
Table 2. Ratio of the Variance Reduction at Tide Gauges [Var (TG-IB)-Var(TG-model)]/Var(TG-IB), as a Percentage, for HF

\begin{tabular}{|c|c|c|c|c|c|c|c|c|c|}
\hline & 1993 & 1994 & $\begin{array}{r}1995 \\
+\mathrm{W}\end{array}$ & $\begin{array}{l}1995 \\
\text { only }\end{array}$ & & $\begin{array}{l}1997 \\
P+W\end{array}$ & $\begin{array}{l}1998 \\
P+W\end{array}$ & $\begin{array}{l}1999 \\
+\mathrm{W} \\
\end{array}$ & $\begin{array}{r}93- \\
\mathrm{P}+\end{array}$ \\
\hline & 45.1 & 4 & 43.9 & & 4 & 43.2 & 44 & 1.8 & 45.4 \\
\hline & & & & & & & & & \\
\hline & & 2 & & & & & & & 20.3 \\
\hline i & 5 & 5 & & & & & & & \\
\hline & & & & & & & & & 1.4 \\
\hline & 59 & & & & & & & 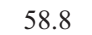 & 57.4 \\
\hline LIs & 37. & & & & 41. & 40 & 45.5 & 0.6 & 41.5 \\
\hline LCo & 20.7 & & & & 29.6 & o & 27.5 & 5 & 27.7 \\
\hline LIs & 14 & & & & & & 10 & 18 & 15.1 \\
\hline & 6 & & & & & & & & \\
\hline & 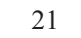 & & & & & & $1 \mathrm{c}$ & 29 & 22.9 \\
\hline nd & 35 & 30.4 & 37.5 & 4.8 & 38.2 & 34.8 & 37.3 & 34.6 & 35.4 \\
\hline
\end{tabular}

The values are averaged over each year named in the column, and over all TG in the spatial region named in each row, for all frequencies. $\operatorname{Var}(\mathrm{x})$ is the variance of $\mathrm{x} . \mathrm{P}+\mathrm{W}$ : simulation forced by pressure and wind; $\mathrm{P}$ only: simulation forced only by atmospheric pressure. GO: Global Ocean; HL: High Latitudes; LL: low latitudes; Co: Coastal TG; Is: Island TG; Atl: Atlantic Ocean; Pac: Pacific Ocean; Ind: Indian Ocean.

as HF). Table 2 shows the percentage of variance reduction after the MOG2D-G correction has been applied. The model allows us to reduce the variance of the corrected signal by a significantly higher percentage than the IB correction alone, although the results depend strongly on location and frequency band.

[17] The pressure-only simulation allows to reduce the TG variance by $7.3 \%$ when compared with the IB correction alone, while the variance reduction reaches more than $50 \%$ when wind stress is included in the forcing. The dynamical character of the ocean response to atmospheric pressure and, the importance of the wind forcing are clearly illustrated.

[18] As expected, the model correction is the most effective at high latitudes : the variance reduction reaches $50.4 \%$ to $56.7 \%$ poleward of $30^{\circ} \mathrm{S}$ and $30^{\circ} \mathrm{N}$, over the period studied. For the equatorial band (between $30^{\circ} \mathrm{S}$ and $30^{\circ} \mathrm{N}$ ), the reduction remains around $17.2 \%-26.5 \%$. Indeed, the high latitudes are very energetic areas where the ocean dynamics have smaller space and time scales, and thus the ocean response to atmospheric forcing is farther from a classical IB response.

[19] Model performances are also better for coastal tide gauges rather than island gauges, which underscores the efficiency of MOG2D-G in shallow water, due to small scales processes and our smaller FE spacing. The effect of the model correction varies depending on the oceanic basin considered, the Atlantic Ocean being the best improved with more than $60 \%$ variance reduction.

[20] Another striking result is the obvious temporal stability of these results (Table 2), during the period 1993-1999. We also notice a clear improvement of the MOG2D-G statistics over time, which is probably explained by improvements in the ECMWF model over the same period. Note that 1996-1999 gives better variance reduction than 1993-1995.

\section{Comparison to Bottom Pressure Records}

[21] The MOG2D-G solutions are also compared to 5 bottom pressure records from the GLOUP database (cf. Figure 2), in order to evaluate the model in the deeper ocean. The observed time series of bottom pressure are
Table 3. Residual Signal Variance at BPR Sites for HF $\left(\mathrm{cm}^{2}\right)$

\begin{tabular}{lccccc}
\hline longitude & latitude & Obs. & Obs-IB & $\begin{array}{c}\text { Obs-model } \\
\text { P only }\end{array}$ & $\begin{array}{c}\text { Obs-model } \\
\mathrm{P}+\mathrm{W}\end{array}$ \\
\hline-57.502 & -56.837 & 108.44 & 6.55 & 6.43 & 4.02 \\
-36.012 & -31.998 & 27.54 & 6.57 & 6.5 & 5.52 \\
-54.715 & -60.85 & 86.1 & 3.97 & 3.13 & 1.73 \\
-56.355 & -58.363 & 90.63 & 6.35 & 6 & 4.64 \\
-58.392 & -54.943 & 93.34 & 3.93 & 4.06 & 3.63 \\
\multicolumn{7}{l}{ Variance reduction compared to }
\end{tabular}

IB correction:

Obs: total variance; Obs-IB: residual variance after IB correction; Obsmodel $\mathrm{P}$ only: residual variance after pressure-alone forced model correction; Obs-model $\mathrm{P}+\mathrm{W}$ : residual variance after pressure and wind forced model correction.

compared with the model sea level minus IB. Table 3 gives the MOG2D-G performances at these sites for the year 1995. For the HF, the pressure forced model induces a 5.2\% reduction of the residual variance compared to the non corrected signal, while the model forced by pressure and wind induces a greater reduction of $29.1 \%$. The model efficiency as well as the strong wind effects are thus highlighted here for the open ocean.

\section{Comparison to Altimetry}

[22] MOG2D-G elevations are used to correct the altimetric sea level data. This approach allows us to evaluate the interest of such a correction for altimetry. The T/P crossovers (CO) were used, with the classical geophysical and instrumental corrections. We removed tides from the $\mathrm{CO}$ time series, by simple harmonic analysis of the 19 main tidal frequencies (M2, S2, N2, K2, K1, O1, P1, 2N2, MU2, NU2, L2, T2, M4, MS4, S1, Q1, OO1, J1, N4). The map given in Figure 3 shows the global variance reduction, obtained when applying the model to correct $\mathrm{CO}$ data, compared to the simple IB correction. As expected, the greater variance reductions (yellow and red colours) are mainly located in some high latitudes deep ocean areas (south-east and north of Pacific, south-west of Australia, south Atlantic) and on continental shelves. The deep ocean red spots on Figure 3 correspond to the areas where the ocean dynamic response to atmospheric forcing has a strong variability, which is induced by a resonant phenomena due

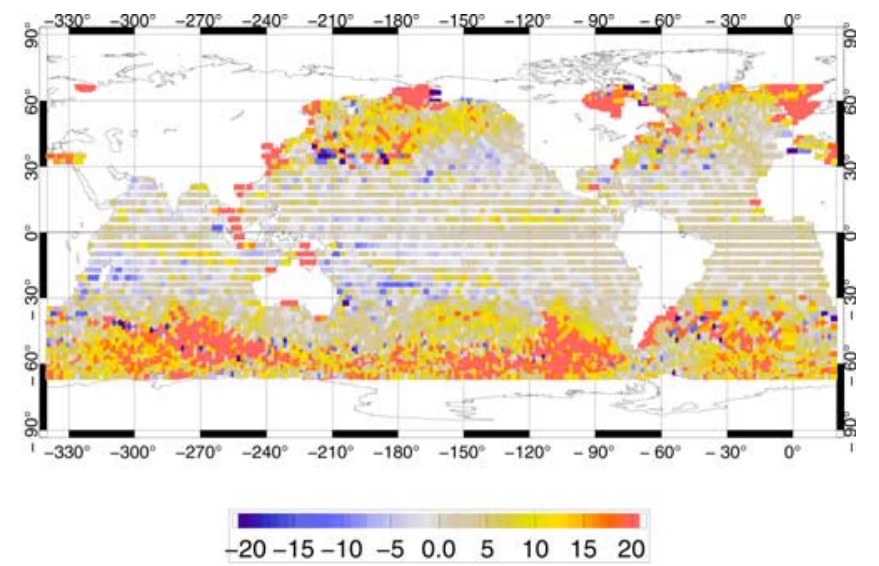

Figure 3. $\operatorname{Var}(\mathrm{CO}-\mathrm{IB})-\operatorname{Var}(\mathrm{CO}-\mathrm{model})$ for the year 1999 in $\mathrm{cm}^{2}$, with the model MOG2D-G forced by atmospheric pressure and wind. 
Table 4. Ratio of the Variance Reduction at CO [Var (CO-IB)$\operatorname{Var}(\mathrm{CO}-\mathrm{model})] / \operatorname{Var}(\mathrm{CO}-\mathrm{IB})$, as a Percentage

\begin{tabular}{|c|c|c|c|c|c|c|c|c|c|}
\hline & $\begin{array}{c}1993 \\
\mathrm{P}+\mathrm{W}\end{array}$ & $\begin{array}{c}1994 \\
\mathrm{P}+\mathrm{W}\end{array}$ & $\mathrm{P}+\mathrm{W}$ & $\begin{array}{c}1995 \\
\text { P only }\end{array}$ & $\begin{array}{c}1996 \\
\mathrm{P}+\mathrm{W}\end{array}$ & $\begin{array}{c}1997 \\
\mathrm{P}+\mathrm{W}\end{array}$ & $\begin{array}{c}1998 \\
P+W\end{array}$ & $\begin{array}{c}1999 \\
\mathrm{P}+\mathrm{W}\end{array}$ & $\begin{array}{l}93- \\
\mathrm{P}+ \\
\end{array}$ \\
\hline & & 11.2 & & & 8 & 13.9 & 14.5 & 13.5 & 12.8 \\
\hline & & & & & & & & & \\
\hline & & & & & & & & & \\
\hline & 35. & & & & & & & & \\
\hline & & & & & & & & & 9.3 \\
\hline & 30.3 & & & & & & & & 8.5 \\
\hline & 7 & & & & & & & & 0.8 \\
\hline L & 22. & 26.2 & & & & & 20 & & 24.3 \\
\hline LLD & 0.5 & 1 & & & & & -0.1 & & 0.7 \\
\hline & & & & & & & & & 3.6 \\
\hline & 1( & & & & & & & & \\
\hline Ind & 7 & 8 & 11.8 & 1.4 & 13.6 & 12.3 & 9 & 11.1 & 10.4 \\
\hline
\end{tabular}

The values are averaged over all years named in the column, and over all $\mathrm{CO}$ in the spatial region named in each $\operatorname{row} . \operatorname{Var}(\mathrm{x})$ is the variance of $\mathrm{x} . \mathrm{P}+$ W: simulation forced by pressure and wind; P only: simulation forced only by atmospheric pressure. GO: Global Ocean; HL: High Latitudes; LL: low latitudes; Sh: shallow waters, depth less than $1000 \mathrm{~m}$; D: Deep ocean, deeper than $1000 \mathrm{~m}$.

to closed contours of $\mathrm{f} / \mathrm{h}$ in the southern ocean [Fukumori et al., 1998].

[23] Table 4 summarises the means of these variance reduction over the period 1993 to 1999 and for different geographic areas. The striking pattern is again the difference between the high and low latitude response: the low latitudes band (between $30^{\circ} \mathrm{S}$ and $30^{\circ} \mathrm{N}$ ) has a lower energy level and the model correction remains very closed to the IB one. At high latitudes (poleward of $30^{\circ} \mathrm{N}$ and $30^{\circ} \mathrm{S}$ ), the signal is more energetic and has smaller characteristic scales; here the model correction is the most relevant. In this last area, the pressure only forcing simulation induces a global variance reduction of only $1.7 \%$ for the year 1995 , while the pressure plus wind forcing reduces the variance by $15-16 \%$ over the $1993-1999$ period. The dominant effects of wind forcing are thus corroborated here.

[24] Once again, the model performs extremely well in shallow waters (depths less than $1000 \mathrm{~m}$ ), with a variance reduction of $34-39 \%$ compared with $6-11 \%$ in the deep ocean. This result points out the efficiency of the refined finite element grid in shallow seas. Note that the MOG2D-G performances at T/P CO remain very stable over the 7 years studied, with a clear improvement after 1995.

\section{Conclusion}

[25] The relevance of our model is to predict the HF global barotropic response to fast and large scale atmospheric forcing, and thus to correct this aliased signal in both the altimetric data and the in situ measurements.

[26] Our results are coherent with earlier studies ([Stammer et al., 2000], [Tierney et al., 2000] and [Hirose et al., 2002]); MOG2D-G induces large variance reductions when applied to observations (TG, BPR, T/P CO). The model correction allows to reduce the variance of the corrected signal by $52.9 \%$ at tidal gauges and nearly $16 \%$ at high latitudes for T/P CO. This variance reduction depends strongly on the frequency band and the locations: high latitudes, continental shelf areas and shallow waters are the best improved. As expected, the low energy equatorial band remains weakly affected by the barotropic model correction. The dominant influence of the wind forcing appears clearly in this study (year 1995 in Tables 2, 3, and 4).

[27] For altimetric purposes, we suggest that only the aliased HF model correction be used (to preserve the wind forced barotropic motions with periods longer than a few days). Various model parameters (including better dissipation processes, and a higher resolution mesh) are still being developed to improve the model.

\section{References}

ECMWF, European Centre for Medium-Range Forecasts Model, ECMWF research manual, 1991.

Egbert, G. D., and R. D. Ray, Significant dissipation of tidal energy in the deep ocean inferred from satellite altimeter data, Nature, 405, 2000.

Fukumori, I., et al., Nature of global large-scale sea level variability in relation to atmospheric forcing: A modeling study, JGR, 103, 5493$5512,1998$.

Gill, A. E., Atmosphere-Ocean Dynamics, vol. 30, 1982.

Hellereman, S., and Rosenstein, Normal wind stress over the world ocean with error estimates, JPO, 13, 1093-1105, 1983.

Hirose, N., et al., High frequency barotropic response to atmospheric disturbances: Sensitivity to forcing, topography, and friction, $J G R, 106(\mathrm{C} 12)$, 2002.

Jayne, S. R., and L. C. St. Laurent, Parameterizing Tidal Dissipation over Rough Topography, GRL, 28(5), 811, 2001.

Lefèvre, F., et al., FES99: A Global Tide Finite Element Solution Assimilating Tide Gauge and Altimetric Information, JAO Tech., 19, 2002.

Lyard, F., The Tides in the Arctic Ocean from a Finite Element Model, JGR, 102(C7), 15,611-15,638, 1997.

Lynch, D. R., and W. G. Gray, A wave equation model for finite element tidal computations, Computers and fluids, 7, 207-228, 1979.

Mathers, E. L., Sea level response o atmospheric pressure and wind forcing in the global deep ocean, PhD Thesis, University of Liverpool, 2000.

Ponchaut, F., et al., An analysis of the tidal signal in the WOCE sea level dataset, JAOT, 18, 2001.

Ponte, R. M., et al., Sea level response to pressure forcing in a barotropic numerical model, JPO, 1043-1057, 1991.

Ponte, R. M., Non equilibrium response of the global ocean to the 5-day Rossby-Haurwitz wave in atmospheric surface pressure, 1997.

Ray, R. D., A global ocean tide model from Topex/Poseidon altimetry: GOT99.2, NASA Technical Memo. 209478, Goddard Space Flight Center, Greenbelt, 58 pp., 1999.

Smagorinsky, J., General circulation experiments with the primitive equations, Monthly Weather Review, 1963.

Stammer, D., et al., De-aliasing of global high frequency barotropic motions in altimeter observations, GRL, 27(8), 1175, 2000.

Tierney, C., et al., Short-period oceanic circulation: implications for satellite altimetry, GRL, 27(9), 1255-1258, 2000.

Wunsch, C., and D. Stammer, Atmospheric loading and the oceanic inverted barometer effect, Reviews of Geophysics, 35, 1997.

L. Carrère and F. Lyard, LEGOS, 18 av. Ed. Belin 31401, Toulouse, France. (carrere@notos.cst.cnes.fr; florent.lyard@cnes.fr) 